\title{
Free flap thrombosis in patients with hypercoagulability: A systematic review
}

\author{
Johannes Albert Biben, Parintosa Atmodiwirjo \\ Division of Plastic Reconstructive and Aesthetic Surgery, Department of Surgery, Dr. Cipto Mangunkusumo National Referral Hospital, \\ Faculty of Medicine Universitas Indonesia, Jakarta, Indonesia
}

Background Even with satisfactory anastomosis technique and adequate experience of the surgeon, flap loss due to thrombosis can still occur due to the patient's underlying condition. Patients with hypercoagulability due to etiologies such as malignancy, hereditary conditions, and acquired thrombophilia are among those who could benefit from free flap procedures. This review aimed to evaluate the risk of free flap thrombosis in patients with hypercoagulability and to identify the most effective thromboprophylaxis regimen.

Methods This review was conducted in accordance with the Preferred Reporting Items for Systematic Reviews and Meta-Analyses guideline. The PubMed, Embase, and Cochrane Library databases were explored. Types of free flaps, types of hypercoagulable states, thrombosis prevention protocols, thrombosis complication rates, and flap vitality outcomes were reviewed. Samples from the included studies were pooled to calculate the relative risk of free flap thrombosis complications in patients with hypercoagulability compared to those without hypercoagulability.

Results In total, 885 articles underwent title, abstract, and full-text screening. Six articles met the inclusion criteria. The etiologies of hypercoagulability varied. The overall incidence of thrombosis and flap loss in hypercoagulable patients was $13 \%$ and $10.3 \%$, respectively. The thrombosis risk was two times higher in hypercoagulable patients $(P=0.074)$ than in controls. Thromboprophylaxis regimens were variable. Heparin was the most commonly used regimen. Conclusions Hypercoagulability did not significantly increase the risk of free flap thrombosis. The most effective thromboprophylaxis regimen could not be determined due to variation in the regimens. Further well-designed studies should be conducted to confirm this finding.

Keywords Free tissue flaps / Thrombophilia / Thrombosis

\author{
Correspondence: \\ Johannes Albert Biben \\ Division of Plastic Reconstructive and \\ Aesthetic Surgery, Department of \\ Surgery, Dr. Cipto Mangunkusumo \\ National Referral Hospital, Faculty of \\ Medicine Universitas Indonesia, \\ Jl. Diponegoro no.71, Central Jakarta \\ 10430, Indonesia \\ Tel: $+62-21-3146938$ \\ Fax: +62-21-3146938 \\ E-mail: johannesalbert.b@gmail.com
}

The authors would like to thank Yenny Rosli (University of California, San Francisco School of Medicine) for her contribution to the manuscript preparation.

Received: June 14, $2019 \bullet$ Revised: September 2, $2019 \bullet$ Accepted: September 29, 2019

pISSN: 2234-6163 • elSSN: 2234-6171 • https://doi.org/10.5999/aps.2019.00738• Arch Plast Surg 2019;46:572-579

\section{INTRODUCTION}

Since Buncke and Schulz [1] successfully performed rabbit ear replantation with vascular anastomoses of less than $1 \mathrm{~mm}$ in 1966, microsurgery has developed rapidly. The success rate of free tissue transfer utilizing microsurgical techniques reached
95.9\% in the late 1990s, and microsurgery has since been established as a dependable reconstructive technique [2].

The anastomosis technique and the surgeon's experience are considered to be the two most important factors contributing to the success of free flap procedures. However, even with satisfactory anastomosis technique and adequate experience of the sur- 
geon, flap loss can still occur. Therefore, the failure of free flap procedures is deemed to be multifactorial $[3,4]$. Other factors associated with flap loss are coagulopathy, infection, vasopressors, hypotension, and the type of the flap [4].

Coagulopathy refers to a group of disorders considered to be a potential cause of flap loss that act synergistically with other well-identified factors [4]. Several reports have suggested that patients with a hypercoagulable state due to conditions such as malignancy, hereditary factors, and acquired thrombophilia might not be good candidates for free tissue transfer procedures [5-7]. However, the advance of microsurgical procedures in recent years has led to the rise of free tissue transfer procedures in malignancy-related reconstruction. Hence, it is essential to investigate the relationship between hypercoagulability and the incidence of thrombosis and flap loss in order to ensure effective patient selection and to design suitable thrombosis prevention protocols for free tissue transfer procedures. Previous review articles explored free flap surgery in hypercoagulable patients, but they have been limited to reports presenting a small number of cases without a control group $[5,8,9]$. This study systematically reviewed the risk of thrombosis in patients with hypercoagulability who underwent a free flap procedure and received a thromboprophylaxis regimen. More importantly, this study emphasized the quality of the evidence and the review process to provide a more reliable summary and recommendations.

\section{METHODS}

We conducted this systematic review according to the Preferred Reporting Items for Systematic Reviews and Meta-Analyses (PRISMA) guideline [10]. We investigated studies that involved free tissue transfer procedures performed in patients with any type of hypercoagulable state. Studies were excluded if they did not clearly present thrombosis as a complication or specify the cause of thrombosis. We also excluded case reports, comments, and letters to the editor.

A systematic literature search of the PubMed, Embase, and Cochrane Library databases was performed on April 1, 2018. The search terms used were "free flap," "microsurgery," "hypercoagulable state," "hypercoagulability," "thrombophilia," "anticoagulant," "anticoagulation," "antithrombotic," and "thromboprophylaxis." The search included related terms in the Medline Medical Subject Heading Library. The literature search was limited to human studies.

For this review, we defined a hypercoagulable state as any hereditary or acquired disorder that rendered the patient susceptible to thromboembolism-related complications. This definition included any previous history of thromboembolism, hereditary mutations of coagulation factors, malignancy, and autoimmune disease confirmed by laboratory evidence $[5,9]$. Thrombosis that occurred at the anastomosis site was considered to be a complication.

Title and abstract screening were performed to identify relevant studies. The full text of selected studies was read. Study selection was based on the inclusion criteria and exclusion criteria, according to consensus between the authors. The following data were extracted from the studies: types of free flaps performed, types of hypercoagulable states, thrombosis prevention protocols, thrombosis complication rates, and final flap outcomes. Samples were pooled when appropriate and allocated into two groups: hypercoagulable patients and non-hypercoagulable patients. The thrombosis and flap loss rate in each group were used to calculate the relative risk of thrombosis complications in patients with hypercoagulability. Studies conducted by the same group of authors were screened for data duplication.

We evaluated the quality of the studies included in this review using the Quality Assessment Tool for Case Series Studies provided by National Institute of Health, Department of Health and Human Services of the US Government [11]. The statistical analysis was performed using SPSS version 20.0 (IBM Corp., Armonk, NY, USA). The chi-square test was used to examine the association between hypercoagulability and the occurrence of thrombosis and flap loss.

\section{RESULTS}

Database exploration yielded 885 articles in the PubMed, Embase, and Cochrane database. Title and abstract screening yielded 16 relevant articles. The full text of those studies was subsequently read to assess whether they matched our inclusion and exclusion criteria. Two studies were excluded because they included pedicled flaps $[7,12]$. One study was excluded because it explored the hemostasis profile in failed cases only [4]. Another study was excluded because some of the thrombophilia diagnoses were based only on thrombosis events related to the free flap procedure without a previous history of thromboembolic events or laboratory confirmation [13]. Four case reports, a letter to the editor, and a literature review were excluded $[5,6,8,9$, $14,15]$. Six series of patients that matched the inclusion and exclusion criteria were included in this review (Fig. 1). The quality of the studies included in this review was assessed according to the Quality Assessment Tool for Case Series Studies checklist as shown in Table 1 [16-21].

Wang et al. [16] studied free tissue transfer in patients with various hypercoagulable conditions in whom several free flap modalities were used. A second study conducted by Wang et al. 


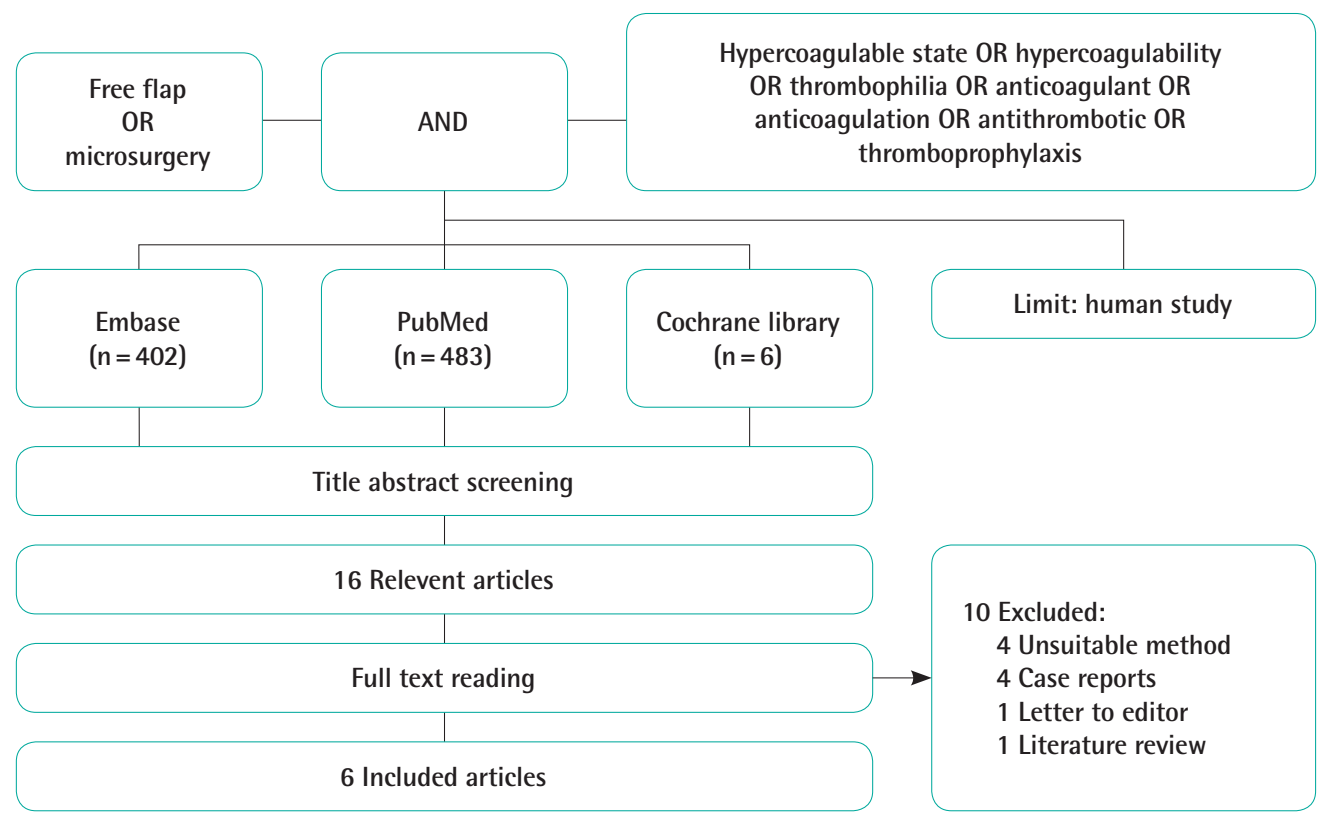

\section{Table 1. Quality assessment of the included studies [12]}

\begin{tabular}{|c|c|c|c|c|c|c|}
\hline Criteria & $\begin{array}{l}\text { Wang et al. } \\
(n=58)[16]\end{array}$ & $\begin{array}{l}\text { Wang et al. } \\
(n=32)[17]\end{array}$ & $\begin{array}{l}\text { Arnljots et al. } \\
(n=103)[18]\end{array}$ & $\begin{array}{l}\text { Olsson et al. } \\
(n=7)[19]\end{array}$ & $\begin{array}{l}\text { Nelson et al. } \\
(n=32)[20]\end{array}$ & $\begin{array}{l}\text { DeFazio et al. } \\
(n=43)[21]\end{array}$ \\
\hline 1. Clearly stated study's question or objective & + & + & + & + & + & + \\
\hline 2. Clearly and fully described study population & + & + & + & + & + & + \\
\hline 3. Consecutive cases & + & + & + & Not stated & + & + \\
\hline 4. Comparable subjects & No comparison & No comparison & Unclear & Unclear & $\begin{array}{l}\text { Retrospective } \\
\text { cohort comparison }\end{array}$ & + \\
\hline 5. Clearly described intervention & + & + & + & + & + & + \\
\hline $\begin{array}{l}\text { 6. Clearly defined, valid, reliable, and consistently } \\
\text { implemented outcome measurement }\end{array}$ & + & + & + & + & + & + \\
\hline 7. Adequate length of follow up & + & + & + & + & + & + \\
\hline 8. Well described statistical method & + & $\begin{array}{l}\text { No statistical } \\
\text { analysis }\end{array}$ & $\begin{array}{l}\text { No statistical } \\
\text { analysis }\end{array}$ & + & + & + \\
\hline 9. Well described results & + & + & - & + & + & + \\
\hline
\end{tabular}

[17] (the same group of researchers) explored free flap procedures performed in hypercoagulable patients with collagen vascular disorders. We examined the cases presented in these two studies and found no subject duplication. Both studies involved the same group of surgeons performing free tissue transfer procedures. Arnljots et al. [18] explored the relationship between hypercoagulability with activated protein $\mathrm{C}$ resistance and the occurrence of free flap loss. Olsson et al. [19] investigated hypercoagulability in patients with malignancy who underwent free flap procedures. Nelson et al. [20] studied anticoagulation protocols used in free flap procedures in hypercoagulable patients. They included a group of subjects that were previously analyzed by Wang et al. $[16,20]$. These subjects were not in- cluded in the pooled data to prevent duplication. DeFazio et al. [21] conducted a study of free tissue transfer procedures for lower extremity reconstruction. Some of their subjects had a hypercoagulable condition. These studies and their findings are outlined in Table 2.

Based on the pooled data, the incidence of thrombosis in hypercoagulable patients was $13 \%$, while the incidence of flap loss was $10.3 \%$. In the non-hypercoagulable group, the incidence of thrombosis and flap loss was $6.3 \%$ and $4.5 \%$, respectively. There was no statistically significant difference in the thrombosis rate $(\mathrm{P}=0.074)$ or flap loss rate $(\mathrm{P}=0.084)$.

All subjects in each study received a thromboprophylaxis regimen regardless of their hypercoagulability status. Various proto- 


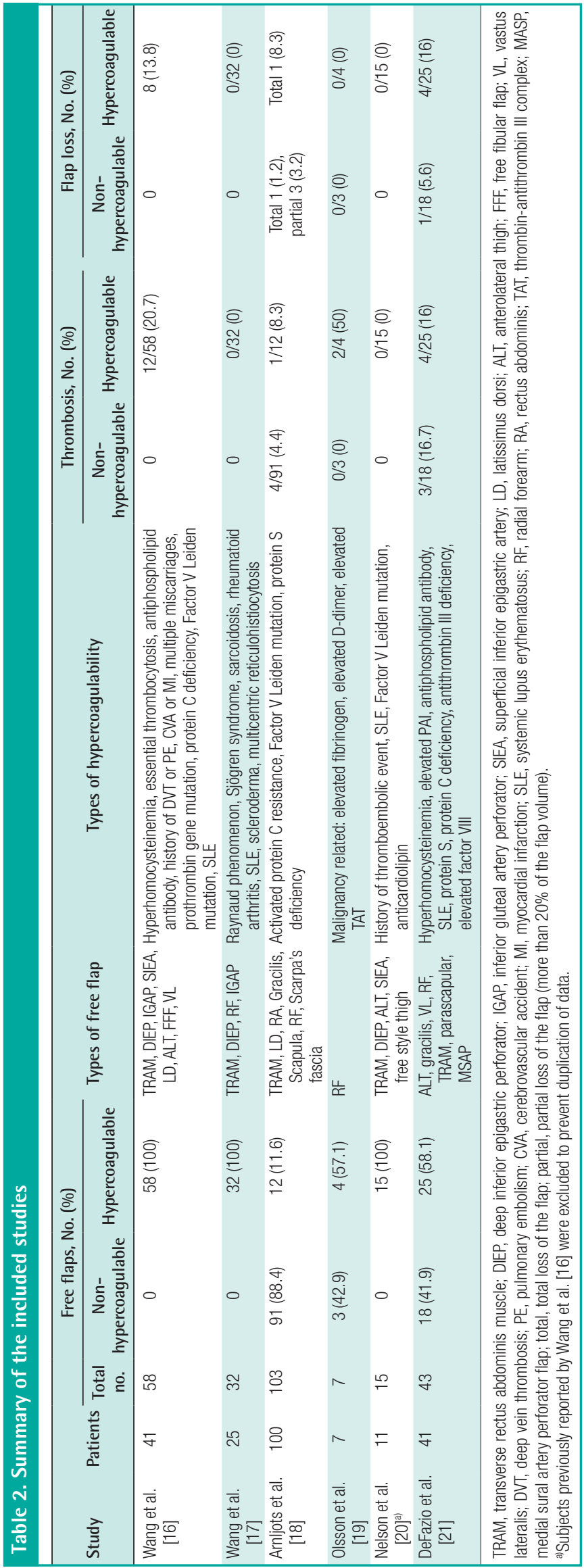

cols were employed in these studies. Heparin was the most common agent used. Wang et al., $[16,17]$ in both of their studies, utilized four main thromboprophylaxis protocols according to each surgeon's routine practice. All of the protocols involved administration of either low-molecular-weight or unfractionated heparin, with or without the addition of aspirin. Some patients who underwent evaluations by hematologists used additional measures, such as clopidogrel, warfarin, and temporary cessation of estrogen receptor antagonist treatment $[16,17]$. No preoperative thromboprophylaxis was given unless advised by the hematologist. Arnljots et al. [18] and Olsson et al. [19] employed preoperative and postoperative thromboprophylaxis using heparin and dextran. Arnljots et al. [18] added dextran and intravenous heparin intraoperatively. Nelson et al. [20] proposed a novel thromboprophylaxis regimen protocol based on a preoperative thrombophilia workup. They mainly used heparin intraoperatively and postoperatively. DeFazio et al. [21] linked the thromboprophylaxis regimen to the occurrence of a thromboembolic event and the decision to perform a salvage procedure. The dose and duration of the thromboprophylaxis regimen varied, as summarized below in Table 3 .

\section{DISCUSSION}

As free tissue transfer comes to be more commonly performed in daily practice, it is expected that microsurgeons will encounter a broader spectrum of patients, including those with thrombophilia. It is widely accepted that the anastomosis technique, the surgeon's experience, and the type of free flap are the most influential factors in free tissue transfer procedures. Nevertheless, thrombophilia or hypercoagulability might pose a considerable problem [4]. In this review, we found six studies that were relevant for determining whether hypercoagulability is related to a higher incidence of free flap thrombosis. We also attempted to evaluate the effectiveness of various thromboprophylaxis regimens. All the studies included were case series, classified as level IV evidence. Some of the recognizable weaknesses of the studies were their retrospective nature, lack of a baseline comparison analysis between groups, historical cohort comparison, and small sample size (Table 1).

The analysis of the pooled data showed that the incidence of thrombosis and flap loss were higher in the hypercoagulable group. The available evidence suggested that there was an overall 2 times higher risk of thrombosis and 2.3 times higher risk of flap loss in patients with hypercoagulability. However, these results were not statistically significant. Therefore, hypercoagulability was not considered a contraindication for free flap procedures. 
Table 3. Summary of thromboprophylaxis regimen across the studies

\begin{tabular}{|c|c|c|c|}
\hline Study & Preoperative thromboprophylaxis & Intraoperative thromboprophylaxis & Postoperative thromboprophylaxis \\
\hline \multirow[t]{4}{*}{ Wang et al. $[16,17]$} & None & None & SQ heparin 5,000 U/8 hr \\
\hline & None & None & $\begin{array}{l}\text { SQ heparin 5,000 U/8 hr, aspirin } 325 \mathrm{mg} \text { for } 2 \text { wk } \\
\text { (started after discharge) }\end{array}$ \\
\hline & None & None & $\begin{array}{l}\text { SQ heparin } 500 \mathrm{U} / 8 \mathrm{hr} \text {, aspirin } 325 \mathrm{mg} \text { for } 2 \text { wk } \\
\text { (started from POD } 1 \text { until } 2 \text { wk) }\end{array}$ \\
\hline & None & IV heparin $300 \mathrm{U} / \mathrm{hr}$ before vessel clamping & $\begin{array}{l}\text { Heparin drip } 300 \mathrm{U} / \mathrm{hr} \text {, aspirin } 81 \mathrm{mg} \text { (started from } \\
\text { POD } 1 \text { until } 2 \text { wk) }\end{array}$ \\
\hline Arnljots et al. [18] & $\begin{array}{l}\text { SQ enoxaparin 2,000-4,000 U (evening } \\
\text { before surgery) }\end{array}$ & $\begin{array}{l}1.000 \mathrm{~mL} \text { Dextran, IV heparin bolus 80-100 } \\
\text { U (during vascular reperfusion) }\end{array}$ & $\begin{array}{l}500 \text { mL Dextran (POD } 2 \text { and 4), SQ enoxaparin } \\
\text { 2,000-4,000 U (POD } 5 \text { until fully ambulant) }\end{array}$ \\
\hline Olsson et al. [19] & $\begin{array}{l}\text { SQ Dalteparin 2,500-5,000 U (evening } \\
\text { before surgery) or 2,500 U (morning) }\end{array}$ & None & SQ Dalteparin 2,500-5,000 U until POD 5-7 \\
\hline Nelson et al. [20] & None & $\begin{array}{l}\text { SQ heparin } 5,000 \mathrm{U} \text { at induction, IV heparin } \\
\text { bolus } 2,000 \mathrm{U} \text { prior to anastomosis, heparin } \\
\text { drip } 500 \mathrm{U} / \mathrm{hr} \text { at time of anastomosis }\end{array}$ & $\begin{array}{l}\text { Heparin drip } 500 \mathrm{U} / \mathrm{hr} \text { (titrated to therapeutic level), } \\
\text { warfarin or enoxaparin (until } 1 \text { mon) }\end{array}$ \\
\hline DeFazio et al. [21] & None & $\begin{array}{l}\text { IV heparin bolus 5,000 U prior to pedicle } \\
\text { division }\end{array}$ & $\begin{array}{l}\text { Depends on thrombotic complication and salvage } \\
\text { procedure: SQ heparin 5,000 U/8 hr, aspirin } 325 \\
\text { mg for } 2 \text { wk, IV heparin bolus } 150 \text { U/kg, heparin } \\
\text { drip } 500 \mathrm{U} / \mathrm{hr}, \mathrm{SQ} \text { enoxaparin for } 2 \text { wk (after } \\
\text { discharge) }\end{array}$ \\
\hline
\end{tabular}

Caution should be taken in interpreting this finding. The thrombosis rate in the hypercoagulable group might have been lower than expected due to the masking effect of the thromboprophylaxis regimen in both the hypercoagulable and non-hypercoagulable groups. While the benefit of anticoagulants in patients without hypercoagulability is still questioned, some evidence has confirmed its role in hypercoagulable patients $[8,9,20]$. This might have led to a lower thrombosis and flap loss rate in the hypercoagulable group, with a consequent impact on the statistical analysis. Since this possibility cannot be excluded, hypercoagulable patients should be identified and treated properly.

The types of hypercoagulability itself might influence the risk of thrombosis, as there were varying rates of thrombosis across the studies. Based on the existing literature, different types of thrombophilia have different risk profiles for thrombosis [22]. For instance, protein $S$ and protein $C$ deficiency had a higher relative risk of venous thromboembolism compared to Factor $\mathrm{V}$ Leiden thrombophilia [22]. However, no previous study has explored these risk variations in microvascular anastomosis. Thrombosis was encountered as a complication in the study of Wang et al. [16], whereas there were no such cases among the collagen vascular disorder patients analyzed by Wang et al. [17] despite the fact that these two studies had an identical thromboprophylaxis strategy, the same operating surgeons, and similar types of free flaps. In contrast, Olsson et al. [19] documented a $50 \%$ thrombosis rate in patients with malignancy-related hypercoagulability. Alternatively, this discrepancy could have been due to the small sample size.

Numerous studies have questioned the benefit of routine anti- coagulation agent administration in free flap procedures. Some studies have even suggested that the use of high-dose heparin may have detrimental effects [23]. However, anticoagulation use in free tissue transfer candidates with a hypercoagulable state has been recommended $[8,9]$.

All the studies included in this review used anticoagulation agents in highly variable doses, durations, and combinations. Wang et al. [16] and Wang et al. [17] employed four main protocols, using primarily postoperative heparin and aspirin administration, with the addition of intraoperative heparin administration in one protocol. Wang et al. [16] performed a statistical analysis revealing that there was no superior protocol among those studied in terms of preventing thrombosis complications. The same thromboprophylaxis regimens resulted in no thrombosis complications in the study of Wang et al. [17], suggesting that hypercoagulability type might influence the result. Wang et al. [16] reported that involvement of a hematologist in the selection of thromboprophylaxis regimen had a positive correlation with good flap outcomes $(\mathrm{P}=0.064)$. Nelson et al. proposed a new thromboprophylaxis protocol and compared it with one of the protocols previously described by Wang et al. [16]. They showed that using a different thromboprophylaxis regimen might affect the thrombosis rate in hypercoagulable patients [20]. While that result seemed promising, further research is needed to overcome the limitations of that study, which include a retrospective comparison and small sample size. DeFazio et al. [21] applied an algorithmic approach to determine the thromboprophylaxis regimen based on the occurrence of thrombotic complications and the decision to perform a salvage procedure. 
By using this protocol, they managed to obtain a comparable incidence of thrombosis between hypercoagulable and non-hypercoagulable patients. However, the flap loss rate was still unsatisfactory.

Arnljots et al. [18] used a combination of heparin and dextran as preoperative, intraoperative, and postoperative anticoagulants. This study was the only one of the six studies we reviewed that utilized dextran. The most recent studies have indicated that dextran use was related to higher rates of thrombosis and free flap loss [24]. Olsson et al. [19] administered heparin preoperatively and postoperatively.

The timing of thromboprophylaxis regimen administration was also a noteworthy variable. Most of the studies used postoperative thromboprophylaxis. Nelson et al. [20] emphasized the importance of using an intraoperative anticoagulant to prevent the formation of the nidus of thrombosis. Preoperative thromboprophylaxis was not a popular option among the studies. The use of preoperative and intraoperative thromboprophylaxis had to be well managed to prevent blood loss and bleeding-related complications.

Since a hypercoagulable state was frequently found during surgery or after flap failure had occurred [7-9,15], free flap candidates must undergo proper preoperative preparation. A thorough history should be taken to discover any previous history of a hypercoagulable state, venous thromboembolism event, or related family history $[8,9]$. When there is no suspicion of hypercoagulability, it is debatable whether a panel of laboratory examinations should be performed. Some studies have pointed out that hereditary thrombophilia is rare. In addition, laboratory tests are costly and negative results do not necessarily exclude hereditary thrombophilia or other biological risks related to thrombosis $[8,9]$. However, a cost-effectiveness analysis found that a limited panel of laboratory tests (activated protein $\mathrm{C}$ resistance, lupus anticoagulant, hyperhomocysteinemia, protein $\mathrm{S}$ deficiency, and factor VIII excess) was actually more cost-efficient than blind administration of thromboprophylaxis for all patients [25]. Thus, we support the use of limited panel tests in such patients. Calculation of the Caprini score might also help in determining the risk of thromboembolism as a complication even though its efficacy to predict thrombosis in microsurgery procedures has not been evaluated [8].

Given negative results of both history and laboratory tests, the choice of using a thromboprophylaxis regimen can be made based on the surgeon's personal experience and preferences. If there is a positive finding in the patient's history, laboratory panel test, or a high-risk classification based on the Caprini score, then a consultation with a hematologist is recommended to determine the most appropriate thromboprophylaxis regimen.
Fig. 2. Detection and management of hypercoagulability in microsurgery

Proposed algorithm for the assessment of free tissue transfer procedure candidates. ${ }^{\text {a) }}$ Activated protein $\mathrm{C}$ resistance, lupus anticoagulant, hyperhomocysteinemia, protein $\mathrm{S}$ deficiency, and factor VIII excess.

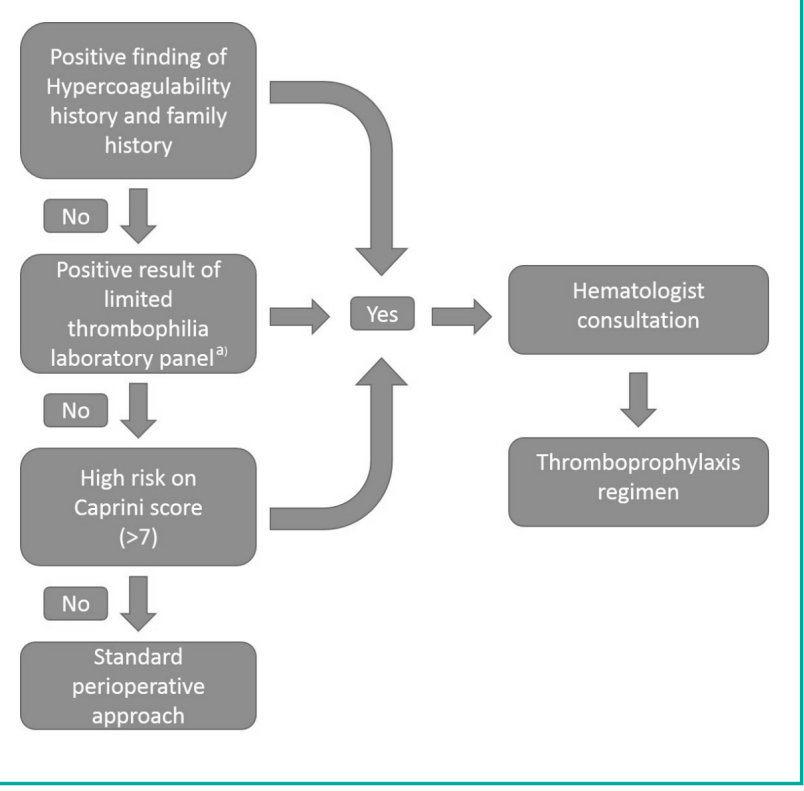

Various studies have suggested that collaboration with a hematologist in the management of these cases might be beneficial $[8,9,16]$. We combined previously reported algorithms and proposed an approach to assess the risk and to manage hypercoagulability in free flap candidates (Fig. 2).

We are aware of the limitations of this review, particularly the fact that all of the studies assessed had a limited level of evidence. Due to the retrospective nature of some studies, we also understand the risks of bias as a limiting factor. In addition, the exclusion of mechanical or technical factors as the etiology of thrombosis complications during re-exploration was an operator-dependent decision, which might have affected the incidence calculations. Lastly, the broad variety of the types of hypercoagulable states and variation in thromboprophylaxis regimens might hinder a clear evaluation of the risk of thrombosis and the role of anticoagulant agents.

A hypercoagulable state did not appear to increase the risk of thrombosis and flap loss in free flap procedures significantly. Stronger evidence is needed to confirm this finding. Therefore, a hypercoagulable state is not a contraindication for free flap procedures, but a thromboprophylaxis regimen should be administered. The most effective thromboprophylaxis regimen could not be determined based on the currently available studies. Well-designed prospective randomized controlled trials of comparable thromboprophylaxis regimens need to be per- 
formed in the future to assess the risk of thrombosis more accurately in patients with various types of hypercoagulability.

\section{NOTES}

\section{Conflict of interest}

No potential conflict of interest relevant to this article was reported.

\section{Ethical approval}

The study was performed in accordance with the principles of the Declaration of Helsinki.

\section{Author contribution}

Conceptualization: Biben JA, Atmodiwirjo P. Data curation: Biben JA, Atmodiwirjo P. Formal analysis: Biben JA, Atmodiwirjo P. Methodology: Biben JA, Atmodiwirjo P. Project administration: Biben JA. Visualization: Biben JA, Atmodiwirjo P. Writing - original draft: Biben JA, Atmodiwirjo P. Writing - review \& editing: Biben JA, Atmodiwirjo P. Approval of final manuscript: all authors.

\section{ORCID}

Johannes Albert Biben https://orcid.org/0000-0002-52242146

Parintosa Atmodiwirjo https://orcid.org/0000-0001-66403310

\section{REFERENCES}

1. Buncke HJ Jr, Schulz WP. Total ear reimplantation in the rabbit utilizing microminiature vascular anastomoses. $\mathrm{Br} \mathrm{J}$ Plast Surg 1966;19:15-22.

2. Khouri RK, Cooley BC, Kunselman AR, et al. A prospective study of microvascular free-flap surgery and outcome. Plast Reconstr Surg 1998;102:711-21.

3. Baumeister S, Follmar KE, Zenn MR, et al. Strategy for reoperative free flaps after failure of a first flap. Plast Reconstr Surg 2008;122:962-71.

4. Davison SP, Clemens MW, Kochuba AL. Anatomy of free flap failures: dissection of a series. Mod Plast Surg 2013;03: 89-95.

5. Srikanthan K, Viswanathan N, Yuen JC. Free-flap failure in thrombophilia: case report and systematic review of the literature. Ann Plast Surg 2013;71:675-81.

6. Khansa I, Colakoglu S, Tomich DC, et al. Factor V Leiden associated with flap loss in microsurgical breast reconstruction. Microsurgery 2011;31:409-12.
7. Sezgin B, Ayhan S, Tuncer S, et al. Hypercoagulability in microvascular breast reconstruction: an algorithmic approach for an underestimated situation.J Reconstr Microsurg 2012; 28:515-20.

8. Pannucci CJ, Kovach SJ, Cuker A. Microsurgery and the hypercoagulable state: a hematologist's perspective. Plast Reconstr Surg 2015;136:545e-552e.

9. Herrera FA, Lee CK, Kryger G, et al. Microsurgery in the hypercoagulable patient: review of the literature. J Reconstr Microsurg 2012;28:305-12.

10. Moher D, Liberati A, Tetzlaff J, et al. Preferred reporting items for systematic reviews and meta-analyses: the PRISMA statement. Int J Surg 2010;8:336-41.

11. National Heart, Lung, and Blood Institute. Quality Assessment Tool for Case Series Studies [Internet]. Bethesda, MD: National Institutes of Health; c2014 [cited 2018 Apr 5]. Available from: https://www.nhlbi.nih.gov/health-topics/study-quality-assessment-tools.

12. Handschel J, Burghardt S, Naujoks C, et al. Parameters predicting complications in flap surgery. Oral Surg Oral Med Oral Pathol Oral Radiol 2013;115:589-94.

13. Senchenkov A, Lemaine V, Tran NV. Management of perioperative microvascular thrombotic complications: the use of multiagent anticoagulation algorithm in 395 consecutive free flaps. J Plast Reconstr Aesthet Surg 2015;68:1293-303.

14. Endara M, Nahabedian M. Free flap breast reconstruction in the hypercoagulable patient with a concomitant bleeding diathesis. Plast Reconstr Surg 2013;132:180e-181e.

15. Davison SP, Kessler CM, Al-Attar A. Microvascular free flap failure caused by unrecognized hypercoagulability. Plast Reconstr Surg 2009;124:490-5.

16. Wang TY, Serletti JM, Cuker A, et al. Free tissue transfer in the hypercoagulable patient: a review of 58 flaps. Plast Reconstr Surg 2012;129:443-53.

17. Wang TY, Serletti JM, Kolasinski S, et al. A review of 32 free flaps in patients with collagen vascular disorders. Plast Reconstr Surg 2012;129:421e-427e.

18. Arnljots B, Soderstrom T, Svensson H. No correlation between activated protein $\mathrm{C}$ resistance and free flap failures in 100 consecutive patients. Plast Reconstr Surg 1998;101:18503.

19. Olsson E, Svartling N, Asko-Seljavaara S, et al. Activation of coagulation and fibrinolysis during reconstructive microsurgery in patients with cancer. Microsurgery 2001;21:208-13.

20. Nelson JA, Chung CU, Bauder AR, et al. Prevention of thrombosis in hypercoagulable patients undergoing microsurgery: a novel anticoagulation protocol. J Plast Reconstr Aesthet Surg 2017;70:307-12. 
21. DeFazio MV, Hung RW, Han KD, et al. Lower extremity flap salvage in thrombophilic patients: managing expectations in the setting of microvascular thrombosis. J Reconstr Microsurg 2016;32:431-44.

22. Moll S. Thrombophilia: clinical-practical aspects. J Thromb Thrombolysis 2015;39:367-78.

23. Pan XL, Chen GX, Shao HW, et al. Effect of heparin on prevention of flap loss in microsurgical free flap transfer: a meta- analysis. PLoS One 2014;9:e95111.

24. Motakef S, Mountziaris PM, Ismail IK, et al. Emerging paradigms in perioperative management for microsurgical free tissue transfer: review of the literature and evidence-based guidelines. Plast Reconstr Surg 2015; 135:290-9.

25. Bowman KG, Carty MJ. Flap complications and thrombophilia: an evidence-based model and cost analysis for preoperative screening. Eplasty 2011;11:e32. 